\title{
Single production of vectorlike quarks with large width at the Large Hadron Collider
}

\author{
Alexandra Carvalho, ${ }^{1}$ Stefano Moretti, ${ }^{2,3,4}$ Dermot O’Brien, ${ }^{2,3}$ Luca Panizzi, ${ }^{5,2}$ and Hugo Prager ${ }^{6,2,3}$ \\ ${ }^{1}$ NICPB, Akadeemia tee, 10143 Tallinn, Estonia \\ ${ }^{2}$ School of Physics and Astronomy, University of Southampton, Highfield, \\ Southampton SO17 1BJ, United Kingdom \\ ${ }^{3}$ Particle Physics Department, Rutherford Appleton Laboratory, Chilton, Didcot, \\ Oxon OX11 OQX, United Kingdom \\ ${ }^{4}$ Physics Department, CERN, CH-1211, Geneva 23, Switzerland \\ ${ }^{5}$ Dipartimento di Fisica, Università di Pisa and INFN, Sezione di Pisa, \\ Largo Pontecorvo 3, I-56127 Pisa, Italy \\ ${ }^{6}$ Theoretische Physik 1, Universität Siegen, Walter-Flex-Straße 3, D-57068 Siegen, Germany
}

(Received 28 May 2018; published 20 July 2018)

\begin{abstract}
Vectorlike quarks (VLQs) are predicted by several theoretical scenarios of new physics and having colour quantum numbers they can copiously be produced at the Large Hadron Collider (LHC) so long that their mass is within the testable kinematic regime of this machine. While it would be convenient to assume that such objects are narrow and can be treated in the so-called narrow width approximation (NWA), this is not always possible: non-minimal new physics scenarios can predict VLQs with potentially large couplings and/or exotic decays, which can contribute to generate a large decay width for such extra quarks without being constrained by current bounds. In this analysis we consider single production of VLQs, which is rapidly becoming a channel of choice in experimental searches owing to the ever increasing limits on their mass, and we describe an approach to treat the large width regime which retains to a large extent a degree of model independence. We also consider the role of potentially sizable and model-dependent interference effects between different VLQ production and decay channels as well as between these and the corresponding irreducible background.
\end{abstract}

DOI: 10.1103/PhysRevD.98.015029

\section{INTRODUCTION}

There is no reason why additional heavy quarks may not exist in Nature: clearly, though, these ought to be heavier than the top quark. However, following the discovery of a Higgs boson at the Large Hadron Collider (LHC) [1,2] closely resembling the one embedded in the standard model (SM), the existence of a fourth generation of chiral quarks (i.e., with SM-like $V-A$ structure in gauge boson charged currents) has been disfavored $[3,4] .{ }^{1}$ A possibility for the existence of additional heavy quarks, even in the presence of a SM Higgs sector, is afforded by vectorlike quarks (VLQs), i.e., heavy spin $1 / 2$ states that transform as triplets

\footnotetext{
${ }^{1}$ If the Higgs sector is enlarged with respect to the $\mathrm{SM}$ to contain additional (pseudo)scalar states [5-7], or if more than one heavy quark multiplet is introduced [8], new chiral quarks are allowed.

Published by the American Physical Society under the terms of the Creative Commons Attribution 4.0 International license. Further distribution of this work must maintain attribution to the author(s) and the published article's title, journal citation, and DOI. Funded by SCOAP.
}

under colour but, differently from the SM quarks, their leftand right-handed ( $\mathrm{LH}$ and $\mathrm{RH}$ ) couplings have the same electroweak (EW) quantum numbers.

VLQs are predicted by several theoretical constructs: e.g., models with a gauged flavour group [9-12], nonminimal supersymmetric scenarios [13-18], grand unified theories [19,20]), little Higgs [21,22] and composite Higgs [23-30] models, to name but a few. In most of these frameworks VLQs appear as partners of the third generation of quarks, having an EW coupling to top and bottom quarks. They are accessible at the LHC detectors in a variety of final states [31-35], some of these already explored by the ATLAS and CMS collaborations [36-44].

Up to now, experimental searches have been designed based on a simplified scenario for modelling the VLQ dynamics, assuming that only one new VLQ is present beyond the SM and parameterising its production, cross section $(\sigma)$, and decay, branching ratio (BR), rates using the narrow width approximation (NWA) [36-41], with the exception of $[42,43]$ where large width scenarios were also considered. In reality, both aspects need not be true. On the one hand, most of the aforementioned VLQ models predict in general the existence of a new quark sector, which 
implies the presence of more than just one new colored state. The possibility of reinterpreting experimental data in the context of scenarios with multiple VLQs has been addressed in [45-48]. Furthermore, especially when such states are (nearly) degenerate in mass, significant interference effects amongst VLQs may occur that would alter the overall yield [49]. On the other hand, VLQs can have a large width, both in virtue of their mass (much larger than the top quark one), their couplings and the variety of decay channels they can undergo, including neutral current ones: contributions from large widths can in turn lead to significant effects, also induced by interferences with the irreducible background, onto the sensitivity of current experimental analyses. Analyses of large width effects in the case of VLQ pair production, i.e., even when the VLQ production stage is due to QCD interactions only, have been performed in [50,51] (see also [52,53]).

In this paper we consider singly produced VLQs mixing with either third or light generations of SM quarks. There are crucial differences between pair and single production processes. If VLQs mix with third generation quarks, their pair production is induced solely by model-independent QCD interactions, while if they mix with light generations, a generally subleading (because quark-antiquark induced) EW component contributes as well. For pair production processes, therefore, large width effects only enters by enhancing off-shell contributions and through the presence of topologies which are subdominant in the NWA. Single production processes, on the other hand, are induced by model-dependent EW interactions at the production level already in the NWA. The same interactions also contribute to determine the width of VLQs, and therefore strongly connect large width contributions to the production rate and interference effects.

In order to interpret the limits of an experimental search on a given range of cross sections one must determine the size of the VLQ couplings which generate such cross sections. If the couplings are large, finite width effect and/or interference effects may be not negligible, and it becomes imperative to take them into account in the experimental searches. The exact relation between the EW couplings and those effects is rather model-dependent, resulting on many possibilities for signal modelling. In this paper, we propose strategies for presenting the results of experimental analyses in a simple, though solid, modelindependent framework, allowing a straightforward reinterpretation of experimental data. A similar approach has been already used in its simplest form in Refs. [42,43]. Such strategy should limit the need of performing numerical recasting for the reinterpretation of results: the possibility of having ways to avoid numerical recasting (when possible) is becoming pressing as experimental searches rely more and more upon numerical frameworks which cannot be easily reproduced in tools for phenomenological analyses, such as boosted decision trees or multivariate analyses.
The plan of the paper is as follows. In Sec. II we describe the analytical model-independent parameterisation and we discuss the issues induced by interference contributions. In Sec. III we perform the phenomenological analysis: first, in Sec. III A, we set up our framework, then, in Sec. III B, we undertake a numerical recasting of a CMS search to obtain bounds for VLQs with large width, finally, in Sec. III C, we adopt an analytical parametrization and compare it to numerical results, also discussing how to treat interference in a specific benchmark. In the final section we draw our conclusions.

\section{MODEL-INDEPENDENT PARAMETRIZATION OF THE CROSS SECTION}

Apart from the aforementioned small contamination due to EW interactions in the case of mixing with light quarks, in processes of VLQ pair production, the VLQs are produced through their QCD couplings to the SM gluons and subsequently decay to the SM quarks through couplings induced by their mixing which, together with the mass of the VLQs, are the free parameters of the beyond the SM (BSM) physics scenario under consideration. The advantage, from a phenomenological point of view, of pair production processes is thus given by the fact that the production of VLQs is essentially only dependent upon their mass, as the QCD coupling is a SM parameter unmodified by new physics.

In processes of VLQ single production, in contrast, the same type of couplings which induce the VLQ decays also determine the production rate, i.e., the cross section of single VLQ production processes depends on their couplings to SM quarks, irrespectively of their mixing patterns. The size of such couplings determines for which VLQ mass the cross section of single production becomes dominant with respect to pair-production at a given collider energy. Moreover, such couplings also contribute to the partial width of the VLQ in their decay channels to SM states. In the case of a VLQ with large width, large couplings of VLQs with SM quarks are in general strongly constrained by oblique observables and Higgs couplings $[35,50,54]$, but such constraints apply to minimal scenarios where the VLQ is the only BSM state. Scenarios with multiple VLQs can induce cancellations which allow to enlarge the couplings while evading current constraints [55]. The total width of the VLQ can also be increased by including further decay channels to other new physics states. To perform an analysis of single production of VLQs with large width it is therefore essential to separate the effects which are purely due to the large width from the dependence of the cross section on the VLQ couplings. As we shall see, in the context of our analysis, such a goal can be achieved considering a suitable parametrization which factors out the contributions of different couplings.

But let us now proceed to define the constituent processes of VLQ production in single mode. In essence, 


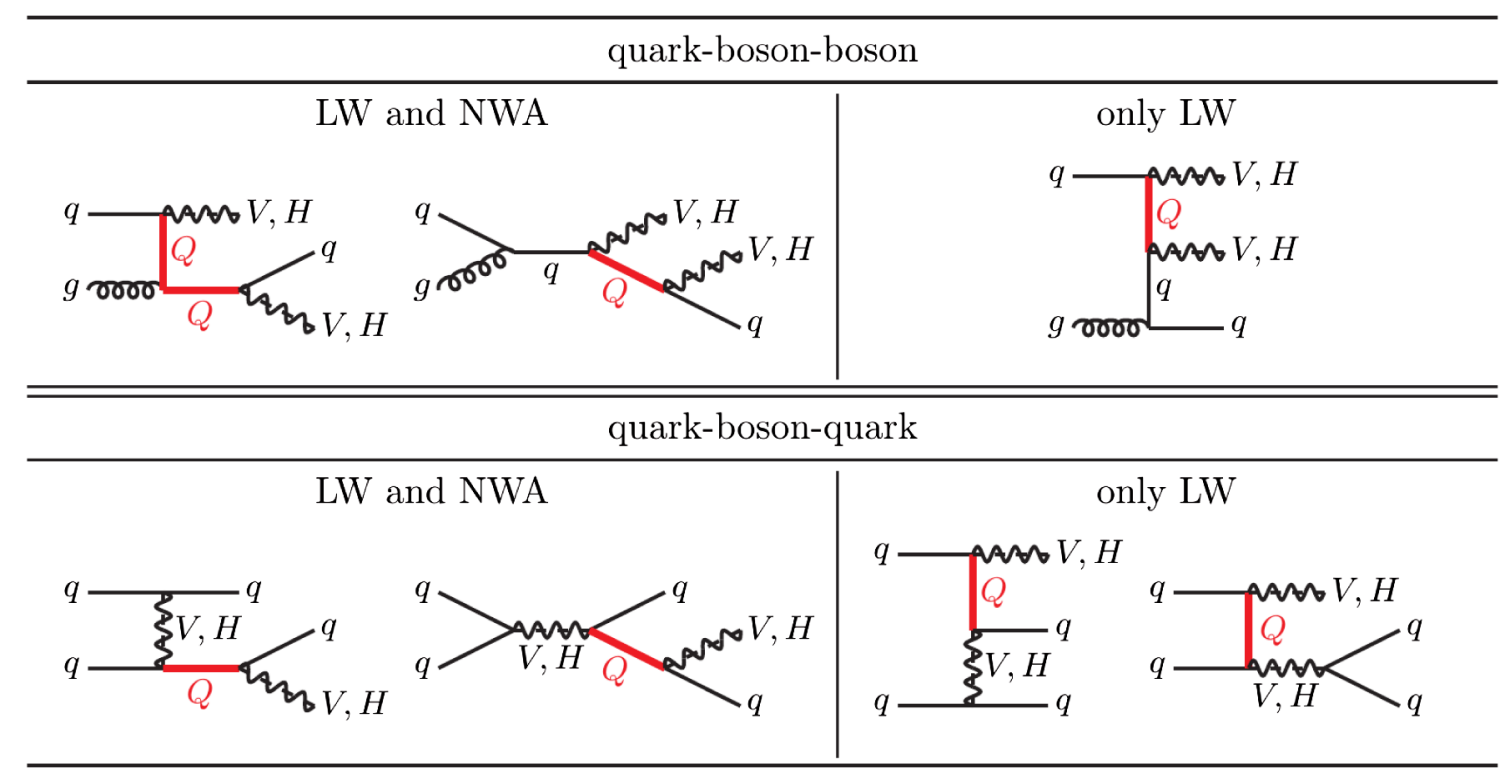

FIG. 1. Complete list of the generic topologies for final states compatible with single VLQ production in the 5FS. Topologies on the left column can be described in both the NWA and LW regimes while topologies on the right column are neglected in the NWA approximation. Here, $V$ represents the $W$ and $Z$ bosons of the SM.

herein, we consider any topology leading to a threeparticle final state made up by SM objects only which contains at least one VLQ propagator. A subtlety, however, arises when deciding upon the initial state. In fact, single production of VLQs can be studied either by considering the proton to be constituted of five quark flavours, the so-called 5-flavor scheme (5FS), or else assuming that bottom quarks are not in the initial state but can only appear from gluon splitting, i.e., the 4-flavor scheme (4FS). Any phenomenological difference between 4FS and 5FS would vanish if it was possible to describe the processes at all orders in QCD perturbation theory. ${ }^{2}$ In our analysis we will work in the 5FS, for which the topologies are represented in Fig. 1. (Notice that some of these only exist in the large width (LW) regime, not in the NWA.) This choice is simply dictated by the preliminary results of Ref. [59] (Contribution 3), which show that, for a specific process and in the NWA, when kinematical distributions of final state objects computed at LO in the 5FS exhibit sizeable differences with respect to those computed at $\mathrm{LO}$ in the 4FS, the distributions obtained through a NLO description of the process are closer in shape to the LO results in the 5FS.

The total cross section $(\sigma)$ can be parametrized by factorizing the BSM couplings considering a reduced quantity, labeled as $\hat{\sigma}$, analogously to what was done in [34]. In the absence of any kind of interference between different topologies, the model-independent $\hat{\sigma}$ depends only on the mass and total width of the VLQ. The factorization

\footnotetext{
${ }^{2}$ For specific details we refer to the wide literature on this subject (see e.g., [56-58] for single top production).
}

of the signal cross section (labeled with the subscript $S$ in the following) can be written as (see Fig. 1):

$$
\sigma_{S}\left(C_{1}, C_{2}, M_{Q}, \Gamma_{Q}\right)=C_{1}^{2} C_{2}^{2} \hat{\sigma}_{S}\left(M_{Q}, \Gamma_{Q}\right),
$$

where $C_{1}$ and $C_{2}$ are the couplings corresponding to the interactions at both sides of the $Q$ propagator, and $\Gamma_{Q}=$ $\Gamma\left(C_{i}, M_{Q}, m_{\text {decays }}\right)$ is the total width of the VLQ, which depends on its mass, the masses of all its decay products and the couplings through which the VLQ interacts with all the particles it can decay to, including (but not exclusively) $C_{1}$ and $C_{2}$.

This parametrization cannot be generalized if interference contributions amongst signal components that contain different coupling content and/or with SM background are non-negligible. In this case the cross sections for the pure signal and its interference contributions with the irreducible background are written as $\sigma_{S}$ and $\sigma_{\mathrm{SB}}^{\mathrm{int}}$, where the second represents the interferences amongst signal $(S)$ topologies or with the SM background $(B)$. In Fig. 2 it is shown an example for the $W t b$ final state: in this case $\sigma_{S}=\sigma_{W}+$ $\sigma_{Z}+\sigma_{W Z}^{\text {int }}$ and $\sigma_{\mathrm{SB}}^{\text {int }}=\sigma_{W B}^{\text {int }}+\sigma_{Z B}^{\text {int }}$ where the subscript of each term represents the bosons propagating in the corresponding topologies. The individual terms of the sum are not necessarily gauge invariant, while the observable quantities $\sigma_{S}$ and $\sigma_{\mathrm{SB}}^{\text {int }}$ are.

The only possible factorization in case interference terms are not negligible is

$$
\begin{aligned}
\sigma_{S}\left(C_{1 \ldots}, C_{2}, M_{Q}, \Gamma_{Q}, \chi_{Q}\right) & =C_{2}^{2} \hat{\sigma}_{S}\left(C_{1 \ldots}, M_{Q}, \Gamma_{Q}, \chi_{Q}\right) \quad \text { and } \\
\sigma_{\mathrm{SB}}^{\text {int }} & =C_{2} \hat{\sigma}_{\mathrm{SB}}^{\text {int }}\left(C_{1 \ldots}, M_{Q}, \Gamma_{Q}, \chi_{Q}\right),
\end{aligned}
$$



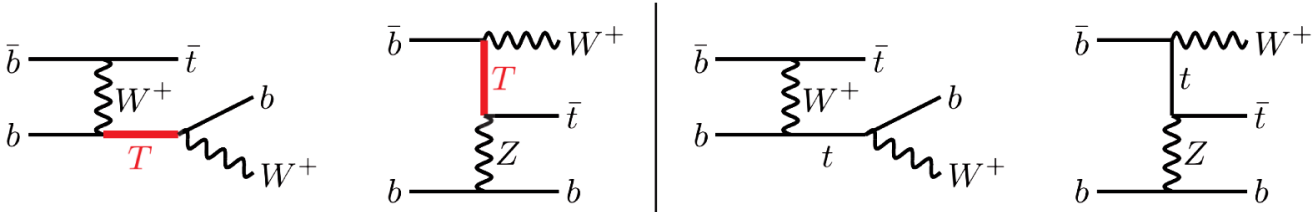

FIG. 2. Example on the left of a subset of interfering topologies in the process $b b \rightarrow b W^{+} \bar{t}$ mediated by a $T$ VLQ and different SM bosons. The diagrams on the right show analogous contributions from the SM background, which interferes with the signal.

where $C_{2}$ is the coupling corresponding to the interaction between $Q$ and the boson in the final state, $C_{1 \ldots}$ are the couplings through which $Q$ interacts with the different virtual bosons and $\chi_{Q}$ the dominant chirality of the VLQ couplings, which can be LH or RH depending on the VLQ representation and for total rates only plays a role in interference terms. As an example, a $T$ singlet will decay through charged current to a bottom quark with a dominantly LH chirality $[34,35]$ whereas a $T$ as part of a doublet will produce a dominantly $\mathrm{RH}$ bottom: the interference with the SM background, for which the bottom quark can only be produced with LH chirality through charged currents, will be different in the two scenarios.

In the limit of negligible interference between different signal topologies, the pure signal contribution can be approximated as:

$$
\sigma_{S}\left(C_{1 \ldots}, C_{2}, M_{Q}, \Gamma_{Q}\right) \sim C_{2}^{2} \sum_{i} C_{1 i}^{2} \hat{\sigma}_{S i}\left(M_{Q}, \Gamma_{Q}\right),
$$

where the potentially gauge-dependent contribution is assumed to be negligible as well.

A further limit is represented by scenarios in which the VLQ interacts exclusively with one SM boson. In this case, besides recovering Eq. (1) for the cross section of the pure signal contribution, the interference with SM background can be written as:

$$
\sigma_{\mathrm{SB}}^{\mathrm{int}}\left(C_{2}, M_{Q}, \Gamma_{Q}, \chi_{Q}\right)=C_{2}^{2} \hat{\sigma}_{\mathrm{SB}}^{\mathrm{int}}\left(M_{Q}, \Gamma_{Q}, \chi_{Q}\right)
$$

Equations (1)-(4) are valid in all width regimes, though their range of applicability is different. The values of $\hat{\sigma}_{S}$ in the parametrization of Eq. (1) can be computed for any possible final state and can be used for a model-independent reinterpretation of the results, under the assumption that the model for which the reinterpretation is made does not contain sizeable interference terms of any kind for the considered final state. Such parametrization, when associated with a proper way to interpret experimental results, discussed below, represents a robust model-independent framework for testing any theoretical scenario predicting VLQs with small or large width against experimental limits in a given channel. This approach has been followed in two CMS analyses $[42,43]$, where $\hat{\sigma}$ was used for an interpretation of experimental results for single production of VLQs with large width. The values of $\hat{\sigma}_{S}$ and $\hat{\sigma}_{\mathrm{SB}}^{\text {int }}$ in
Eqs. (2)-(4), however, contain a further model-dependency, represented by the impossibility to factorize the $\left\{C_{1 \ldots}\right\}$ couplings in a gauge-invariant way. If interference terms are sizeable, this is an unavoidable consequence and the analysis would have in any case a limited reinterpretation applicability.

Both parametrizations can be easily generalized to compute cross sections at NLO in QCD, as the factorization of the EW couplings to derive the $\hat{\sigma}$ 's is not affected by the contribution of radiative QCD effects. A NLO calculation of single production of VLQs with finite width is however beyond the scope of this paper.

\section{PHENOMENOLOGICAL ANALYSIS}

In this section we will provide case studies for the reinterpretation of experimental results of searches for single production of VLQs with finite width, comparing the bounds obtained through a numerical recasting with the results obtained applying the analysis strategies of Sec. II.

\section{A. Benchmarks}

Analogously to what has already been done in the CMS analyses $[42,43]$, we will consider specific and representative scenarios for the interactions of VLQs with the SM states. Couplings between VLQs and SM quarks and bosons are parametrized as:

$c_{Z}=\frac{e}{2 c_{w} s_{w}} \kappa_{Z}, \quad c_{W}=\frac{e}{\sqrt{2} s_{w}} \kappa_{W} \quad$ and $\quad c_{H}=\frac{M_{Q}}{v} \kappa_{H}$,

where $v=246 \mathrm{GeV}$ is the Higgs vacuum expectation value (VEV), $c_{w}$ and $s_{w}$ are the cosine and sine of the weak angle $\theta_{w}$ and $\kappa_{W, Z, H}$ are coupling strengths. In the limit where only one VLQ representation is present in Nature and the particles are narrow the $\kappa$ parameters accurately approximate the sine of the mixing angle between VLQ and SM quark (for a relation between couplings and mixing angles see, e.g., Ref. [54]). In the NWA, if the VLQ is a singlet, the coupling parameters are related as $\kappa_{Z} \simeq \kappa_{H} \simeq \kappa_{W} \simeq \kappa$ while, if the VLQ is within a doublet (and assuming that the Yukawa coupling of the other element of the doublet is zero), the coupling parameters become $\kappa_{Z} \simeq \kappa_{H} \simeq \kappa$ and $\kappa_{W} \simeq 0$. These relations are not satisfied in the large width regime. 
TABLE I. Benchmark points and corresponding coupling relations.

\begin{tabular}{lcccc}
\hline \hline $100 \% W$ & $100 \% Z$ & $100 \% H$ & Singlet-like & Doublet-like \\
\hline$\kappa_{Z}^{q}=\kappa_{H}^{q}=0$ & $\kappa_{W}^{q}=\kappa_{H}^{q}=0$ & $\kappa_{W}^{q}=\kappa_{Z}^{q}=0$ & $\kappa_{W}^{q}=\kappa_{Z}^{q}=\kappa_{H}^{q}$ & $\kappa_{W}^{q}=0$ and $\kappa_{Z}^{q}=\kappa_{H}^{q}$ \\
\hline \hline
\end{tabular}

Minimal extensions of the SM with one unique VLQ representation where the VLQ has large couplings are excluded by other constraints (such as EW precision tests or corrections to SM couplings) [35,50,54]. In our case study the large width regime will be achieved by considering the total width as a free parameter but imposing for VLQ singlets and doublets identical relations between the $\kappa$ parameters as in the NWA. We also consider more extreme scenarios where we assume 100\% couplings of the VLQ with each of the SM bosons. A summary of the benchmarks we consider is reported in Table I. Through this procedure the assumption of a minimal model is relaxed: new physics, in the form of mixings either in the quark or bosonic sectors due to additional representations of VLQs or new gauge bosons or scalar fields, must contribute to generate the relations between couplings.

The event generation has been performed through MADGRAPH5_AMC@NLO $[60,61]$ using the NNPDF3.0 PDF set [62] at LO with $\alpha_{S}=0.118$ and setting the QCD renormalization and PDF factorization scales to the mass of the VLQ. The generated events have been subsequently processed through PYTHIA $8[63,64]$ to include parton showering and hadronization. Finally, the hadronized events have been analyzed through
MadAnalysis 5 [65], which uses Delphes 3 [66] for the emulation of detector effects. The VLQ model used for the simulation was implemented in FEYNRULES [67] to obtain the UFO [68] model format to be used inside MADGRAPH5_AMC@NLO. This model is a slightly modified version of the public model described in [69] where each coupling of the VLQ has been assigned a different label in order to isolate individual interference contributions through the MADGRAPH5_AMC@NLO syntax COUPLING_ORDER_I $\wedge 2==\mathrm{N}$ in the process of diagram generation, where the Ith coupling order is counted $\mathrm{N}$ times in the squared amplitudes, making it possible to generate separately each term of Eq. (2).

It is not within the scope of this paper to investigate the impact on searches due to the choice of the proton scheme. However, as most of the experimental searches performed so far adopt the 4FS (including the one recast in this analysis) a more quantitative discussion about the choice of the flavour number scheme is in order. As an example, in Fig. 3, we show the $p_{T}$ distribution of the leading $b$-jet comparing the $4 \mathrm{FS}$ and $5 \mathrm{FS}$ at LO for a $B$ VLQ mixing with third generation, in different width regimes, for the processes $p p \rightarrow t W j$ and $p p \rightarrow b Z j$. The dependence of the distributions on the width of the VLQ can be sizeable,
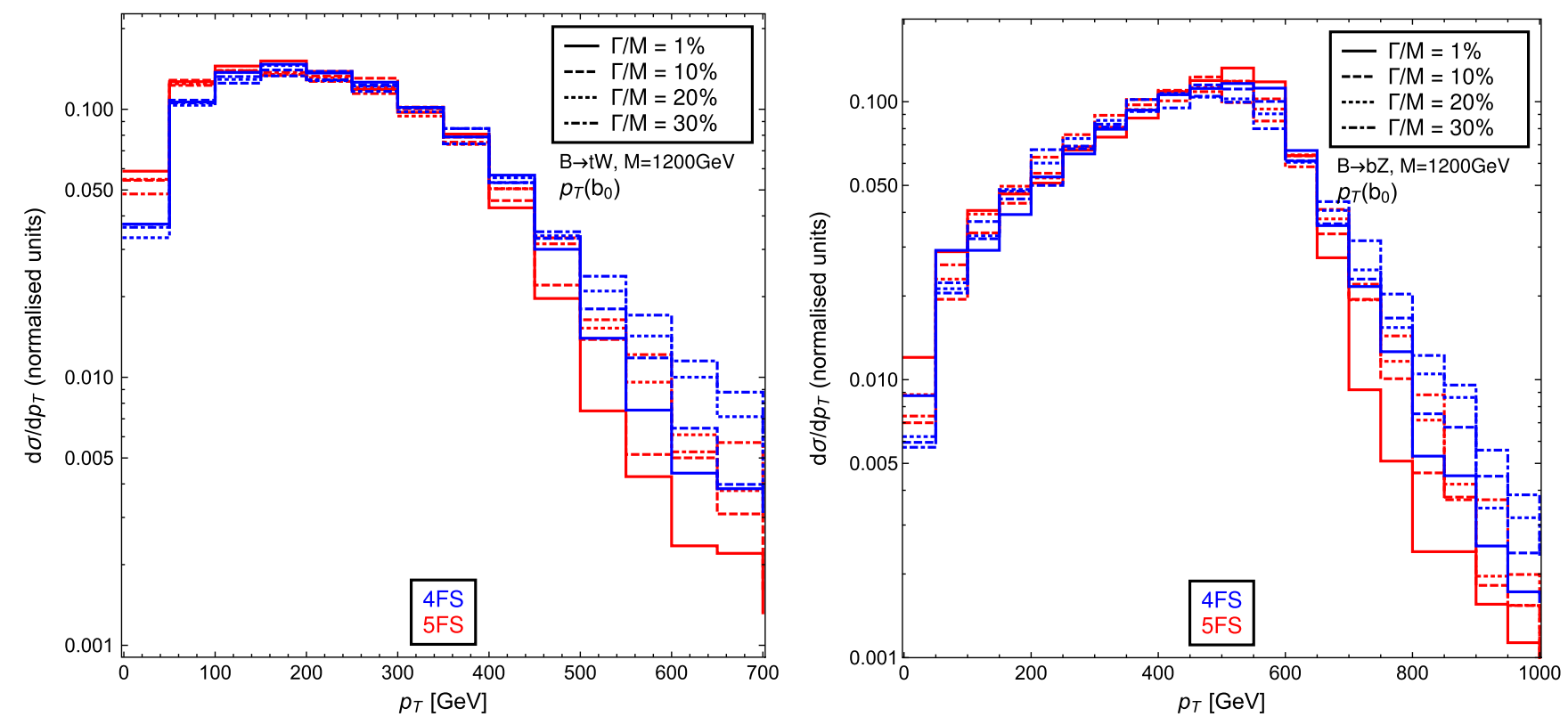

FIG. 3. Distributions of the transverse momentum of the leading $b$-tagged jet for two processes. Left panel: $p p \rightarrow t W j$ (in the 5FS) or $p p \rightarrow t W j b$ (in the 4FS); right panel: $p p \rightarrow b Z j$ (in the 5FS) or $p p \rightarrow b Z j b$ (in the 4FS), with subsequent on-shell decays of the SM states in both cases. The comparison is made for a $B$ mass of $1200 \mathrm{GeV}$ and for different values of the width-over-mass ratio $\left(\Gamma_{Q} / M_{Q}\right)$ of the VLQ. 
thus potentially affecting the efficiency of experimental cuts. The final state $b$-quark is dominantly generated in the $B$ decay and therefore is fairly central in both 5FS and 4FS in any width regime. The width-dependent differences in the shapes are generally of similar size in both $4 \mathrm{FS}$ and $5 \mathrm{FS}$.

The signal cross sections are generally of similar size between 4FS and 5FS in the NWA at LO [59]. We had also checked that in all parameter space we probe the cross sections calculated in the 4FS are always smaller than the ones calculated on the 5FS. Therefore, the interpretation of results in terms of number of events in the 4FS acts as a lower bound to the a more realistic exclusion bound.

\section{B. Recasting and reinterpretation of experimental data}

As a representative analysis for the recast of experimental data, the CMS search CMS-B2G-16-006 [70] has been chosen. This analysis specifically targets a heavy VLQ with charge $+2 / 3$ or $-4 / 3$ decaying into a $b$-quark and a $W$ boson, which is produced singly in association with a light flavour quark and a $b$-quark. Thus, in the 4FS, the considered process is $p p \rightarrow T(\rightarrow W b) \bar{b} j$. The search selects events with one lepton (electron or muon in two different signal regions) and at least two jets, one central ( $b$-tagged) and one forward. A set of kinematical cuts are also imposed, and we refer to [70] for all the relevant details. In the experimental analysis the limits are computed analyzing the shape of the mass of the heavy quark candidate, formed by the vectorial sum of the lepton, the leading central jet, and the neutrino candidate (where the neutrino candidate is reconstructed assuming its $\mathrm{x}, \mathrm{y}$-components to be the missing transverse momentum and that the invariant mass of it summed with the lepton is the $W$ mass). In our case the limits are computed by a simple counting on the signal region. In Table II we compare the efficiencies obtained by our recast implementation with the ones reported by [70] under the same signal assumptions of the experimental search. We stress that, for the purposes of this study, we are not interested in an overly precise reconstruction of the experimental results: our intent is to achieve a realistic analysis framework to demonstrate how to reinterpret experimental data in scenarios where the VLQs have a large width.

We recast this analysis for the large width case by scanning the $\Gamma_{Q} / M_{Q}$ from, e.g., $1 \%$ to $40 \%$ in steps of $5 \%$ and $M_{Q}$ from 600 to $2000 \mathrm{GeV}$ in steps of $100 \mathrm{GeV}$.

TABLE II. Comparison of the experimental and recasted efficiencies for the different signal regions for a $p p \rightarrow T(\rightarrow$ $W b) j b$ signal in the 4FS with $m_{T}=1000 \mathrm{GeV}$ in the NWA.

\begin{tabular}{lclc}
\hline \hline Channel & Electron & Muon & Total \\
\hline Experimental efficiencies & $1.3 \%$ & $1.4 \%$ & $2.7 \%$ \\
Recasting efficiencies & $0.98 \%$ & $1.31 \%$ & $2.29 \%$ \\
\hline \hline
\end{tabular}

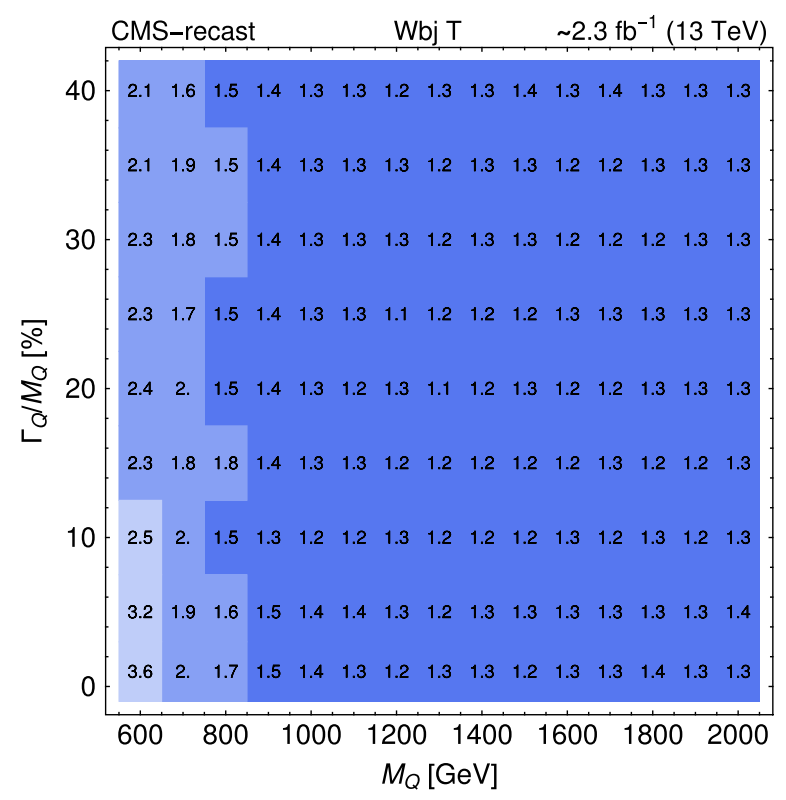

FIG. 4. 95\% CL largest allowed cross sections (in pb) for the $W b j$ final state as a function of $M_{T}$ and of $\Gamma_{T} / M_{T}$ for a VLQ $T$ coupling to the third SM quark generation and decaying $100 \%$ to $b W$.

Four processes will be considered: the original target of the search (a third generation $T$ partner in the $p p \rightarrow W b q$ channel) but also a third generation $B$ partner in the $p p \rightarrow$ $W t t$ channel, and a light generation $T$ and $B$ partner on the $p p \rightarrow W \bar{q} q$ channel (notice that, as we consider the 5FS, a $q$ may also be a $b$ ). For the sake of linearity, we first show the results of the recast in the $p p \rightarrow W b q$ channel. The values of the largest allowed observed cross section at 95\% confidence level (CL) on the $\left(M_{Q}, \Gamma_{Q} / M_{Q}\right)$ plane that we obtain for this channel are shown in Fig. 4.

To interpret the results with the benchmarks of Table I we saturate the values of the VLQ couplings to obtain the needed size of the width for each point in the $\left(M_{Q}, \Gamma_{Q} / M_{Q}\right)$ grid of our scan. The total cross section obtained by saturation is then compared to the experimental limits and it is verified to be excluded or not. In fact, the coupling necessary to fix $\Gamma_{Q} / M_{Q}$ have an effect on the cross section of the process and therefore on the region of exclusion on the $\left(M_{Q}, \Gamma_{Q} / M_{Q}\right)$ plane, using the same data. For instance, trivially, the value of the VLQ couplings will be larger for benchmark points where they interact $100 \%$ in a specific channel than for the singlet- or doublet-like benchmarks, given the contribution from other decay channel(s) in the latter case.

This done in Fig. 5, where we show the value of the $W b j$ signal cross section as a function of the VLQ mass and of its $\Gamma_{Q} / M_{Q}$ ratio, together with the $95 \%$ exclusion line for the $W b j$ final state. It is possible to notice that the NWA region is not excluded while the exclusion range appears as $\Gamma_{Q} / M_{Q}$ increases, thus highlighting the phenomenological impact of the LW regime. The shape of the exclusion 

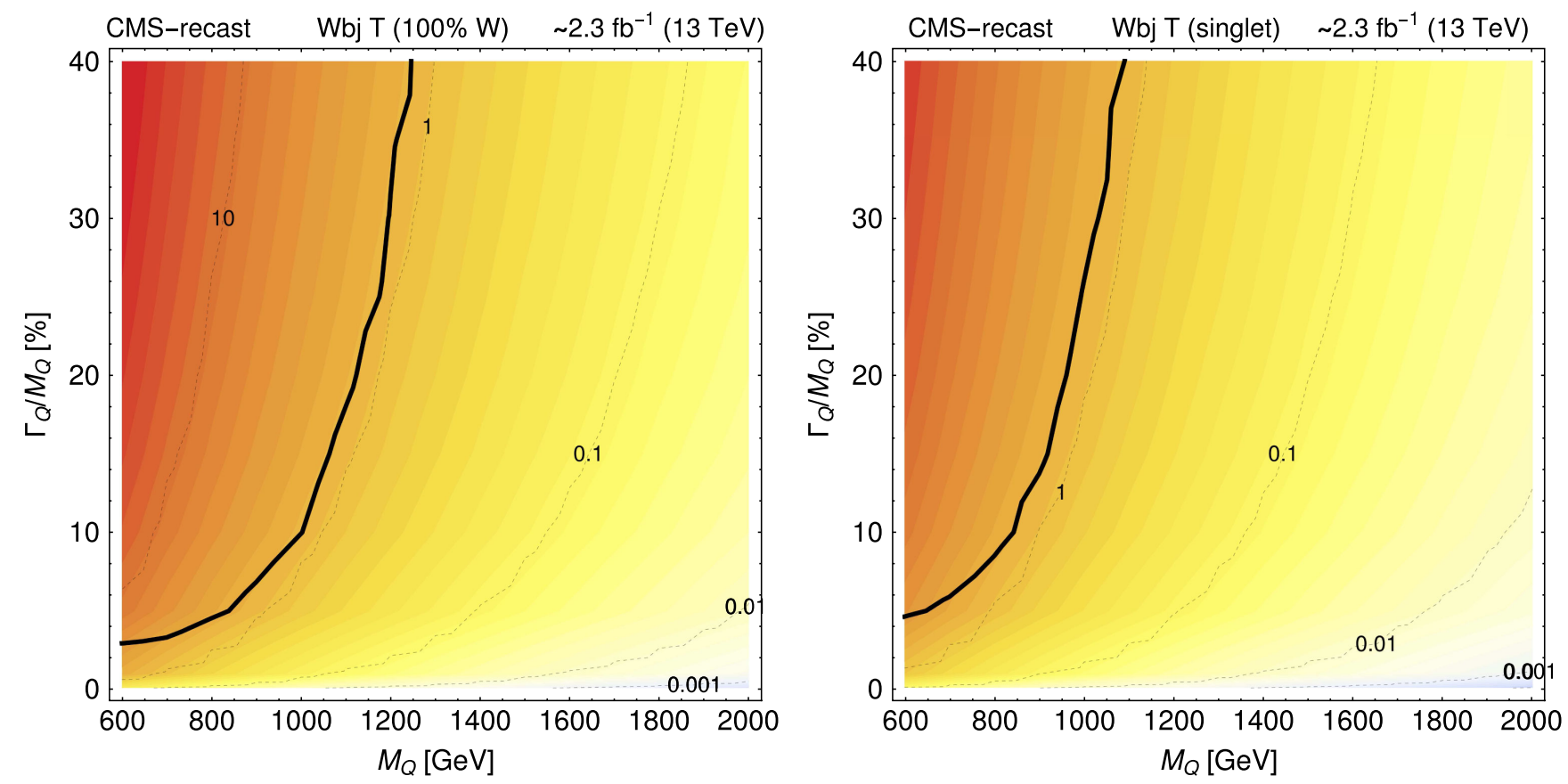

FIG. 5. Cross section (in pb) for the $W b j$ final state as a function of $M_{T}$ and of $\Gamma_{T} / M_{T}$ and $95 \%$ exclusion line (in black) for a VLQ $T$ decaying $100 \%$ to $b W$ (left panel) or singlet-like (right panel).

boundary is a balance between how the theory cross section grows on the $\left(M_{Q}, \Gamma_{Q} / M_{Q}\right)$ plane and the behavior of the signal kinematics. In Fig. 5 we see that the exclusion bounds roughly track the variation of the cross section, with the exclusion line in the region where the cross section is slightly larger than $1 \mathrm{pb}$. Such behaviour is related to the fact that, for the considered CMS search, selection cuts are designed in such a way that the signal efficiencies vary only moderately in the whole plane. In the light of this result, in the following, we will only show results for VLQ coupling which are $100 \%$ to a specific SM boson, the results for singlet- and doublet-like cases being qualitatively analogous to a simple rescaling of the cross section.

In Fig. 6 we show the values of the largest allowed cross section at $95 \% \mathrm{CL}$, together with the exclusion bound, for each point of the grid in the $\left(M, \Gamma_{Q} / M_{Q}\right)$ plane for a VLQ $T$ (top row) or $B$ (bottom row) coupling to the first (top and bottom-left panels) or third (bottom-right panel) SM quark generations. For the scenario where the $B$ couples with the third SM quark generation (bottom-right) the value of the largest allowed cross section is rather large in comparison with the other benchmarks we consider and therefore no exclusion can be made. ${ }^{3}$ In contrast, we do find exclusion regions for the $W j j$ channel mediated by a light generation $T$ or $B$ partner.

\footnotetext{
${ }^{3}$ This can be explained by a combination of two factors: the final state $W t t$ is heavier than the other ones we consider, leading to a much smaller cross section and, since the search is not targeting this specific final state, the signal efficiencies are smaller than for the other channels.
}

Analogously to the $W b j$ case, the exclusion lines in both the $W j j$ scenarios basically follow the increase of the cross section due to the increase of the coupling. However, as the search is not optimized for $W j j$ final states, the largest allowed cross section is much larger than for the $W b j$ case $^{4}$ The fact that we still get a similar exclusion for these final states is due to the higher cross section for the light generation VLQs: PDF effects makes the $b$-initiated processes less likely that the $u$-and $d$-initiated ones, leading to a much larger cross section for the $W j j$ final states. Between the two $W j j$ plot results, we observe a stronger exclusion in the case of the propagation of a $B$ VLQ, rather than a $T$ VLQ, due to the fact that the cross section is larger while the efficiencies are similar in the whole parameter space.

In all the channels we consider the largest allowed cross section decreases when the mass increases but, except for small masses (less than $1 \mathrm{TeV}$ ), this search is not really sensitive to the width variation. The effect of the width on the excluded cross section is larger for $M_{Q} \simeq 600 \mathrm{GeV}$ where it can increase by a factor 4 with $\Gamma_{Q} / M_{Q}$ increasing from the NWA to $\simeq 40 \%$. The overall weak dependence of the results on the VLQ width is related to the fact that the kinematic distributions of the final states are also often weakly affected. The same weak dependence was found in the two CMS single production searches exploring the large VLQ width regime [42,43].

\footnotetext{
${ }^{4}$ For instance, the search we are recasting requires a $b$ quark in the final state, which can only come from the accompanying jet when the VLQ only decays to light quarks.
} 

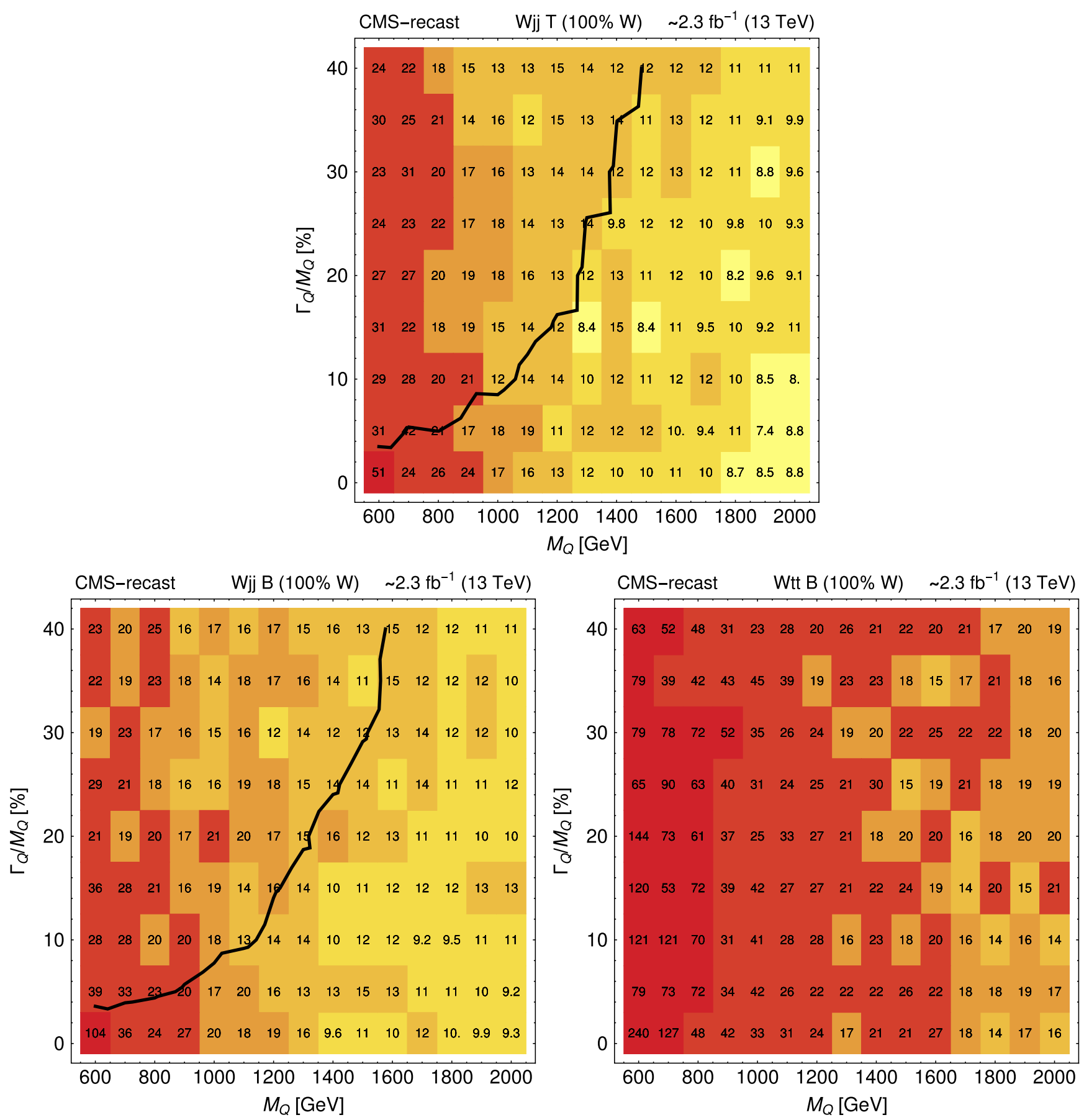

FIG. 6. 95\% CL excluded cross sections (in pb) and exclusion bound (black line) as a function of $M_{Q}$ and $\Gamma_{Q} / M_{Q}$ for a VLQ $T$ (top row) and $B$ (bottom row) coupling to the first (top and bottom-left panels) or third (bottom-right panel) SM quark generation.

\section{Reinterpretation through the reduced cross section $\hat{\sigma}$}

In this section we provide an example of how to interpret experimental data only using the reduced cross section $\hat{\sigma}$ described in Sec. II for a model featuring a VLQ interaction with SM bosons with given couplings, mass and total width. The procedure can be generalized for any search of BSM states which can decay into SM final states, for which the width of the resonance can be considered as a further degree of freedom, complementary to the mass of the BSM state, for the determination of the maximum excluded cross section.

If the interference terms are negligible for the signal topologies, and likewise for the interference between signal and SM background, then the cross section for any process $p p \rightarrow V q q$, where $V$ is any SM boson and $q$ any SM quark, 

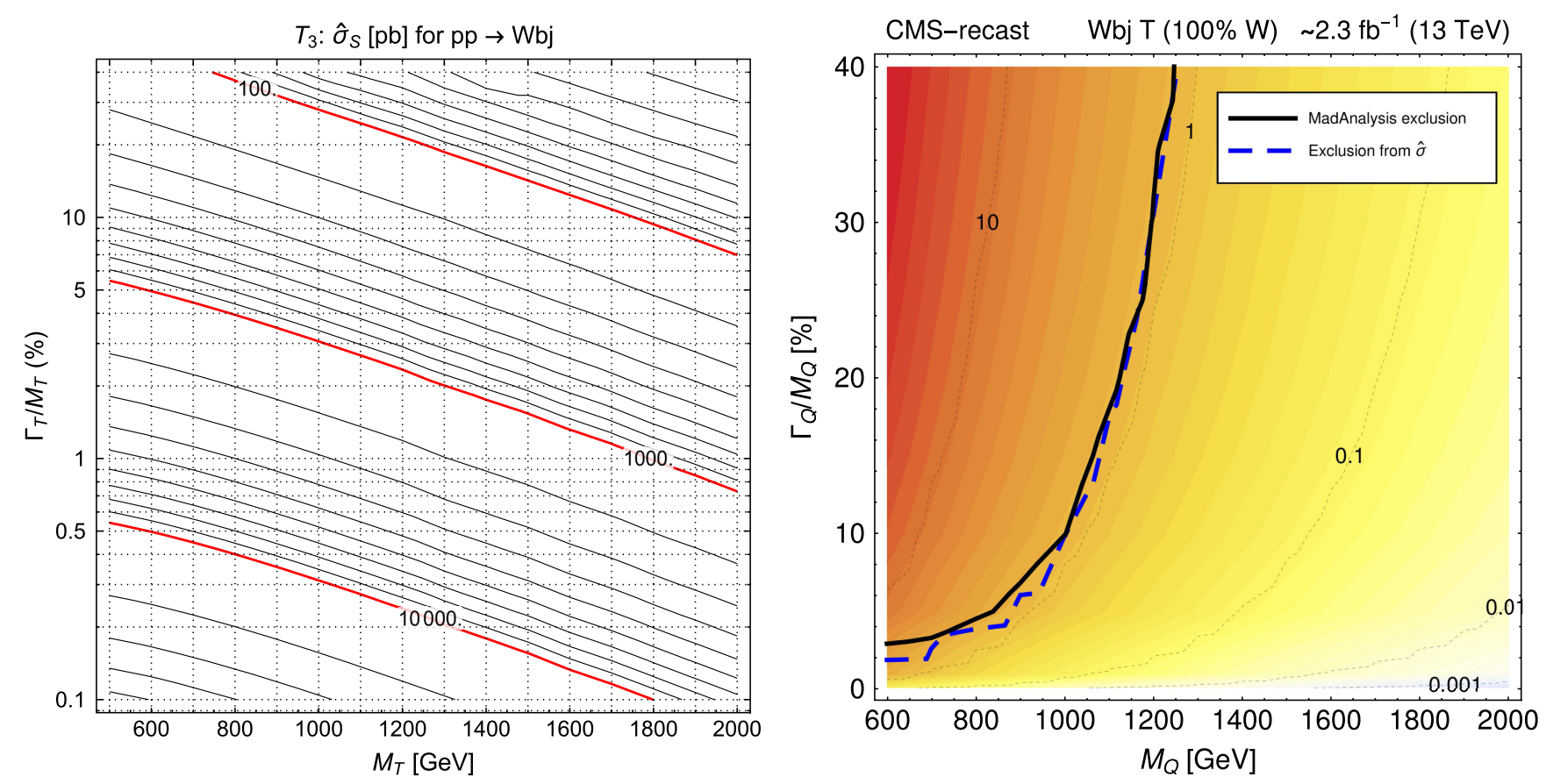

FIG. 7. The $13 \mathrm{TeV} \hat{\sigma}$ values (left) and the signal cross section (right), both given in pb, as a function of $M_{Q}$ and $\Gamma_{Q} / M_{Q}$ for a VLQ $T$ coupling to the third generation shown together with the exclusions line obtained using the $\hat{\sigma}$ data (in dashed blue) and with the original exclusion line (in black, same as Fig. 5), assuming negligible interference contributions from any source.

can be obtained from the $\hat{\sigma}$ cross sections, rescaled with appropriate couplings. By comparing it to the excluded cross sections (minding the model-dependent relation between the couplings and $\Gamma_{Q} / M_{Q}$ ) it is possible to determine whether a specific scenario is excluded by the experimental search without the need of performing a dedicated recast (as it was done to construct Fig. 4). This is possible provided the results of the searches are presented in the $\left(M_{Q}, \Gamma_{Q} / M_{Q}\right)$ plane, as it has been done in the last section and in Refs. $[42,43]$.

In Fig. 7 the $\hat{\sigma}$ 's for the process $p p \rightarrow b W j$ mediated by a $T$ interacting exclusively through charged current are shown on the left panel while on the right panel we report a comparison between the limit obtained through a numerical recasting and the limit obtained by rescaling the $\hat{\sigma}$ values with the coupling needed to obtain the corresponding width of the $T$ in this benchmark scenario. The results are qualitatively very close and allow to identify with good accuracy the excluded region of this benchmark scenario.

\section{Including interference terms for $p p \rightarrow$ Wbj via $T$}

Finally, we consider the role of interference between signal and background for the $p p \rightarrow b W j$ channel with propagation of a $T$ VLQ. This scenario is studied under two hypotheses: (1) $T$ mixing with third SM quark generation and coupling only through charged current, such that new physics allowing for large width can be achieved only by requiring mixing with other new states in the VLQ sector; (2) $T$ can only decay to $W b$ in the SM, but other BSM decay channels (which do not play a role as interference within signal itself) are open, such as, e.g., $T \rightarrow S t$ for a (generic) new BSM scalar $S$. Such assumptions allow us to study the scenario through the parametrization of Eqs. (1) and (4). To perform the analysis for both cases, we start from the fact that, for any specific point, characterized by the mass and $\Gamma_{Q} / M_{Q}$ ratio of the VLQ, the total number of events is composed by different contributions:

$S+B=L\left(\sigma_{S} \epsilon_{S}+\sigma_{S B_{\text {irr }}}^{\text {int }} \epsilon_{S B_{\text {irr }}}^{\text {int }}\right)+B_{\text {irr }+ \text { red }} \equiv L \sigma_{\text {eff }}+B$,

where $L$ is the integrated luminosity, $B_{\text {irr }}$ and $B_{\text {red }}$ are the number of events for the irreducible and reducible background terms respectively (which sum to the total number of background events $B$ ) and $\sigma_{\text {eff }} \equiv \sigma_{S \text {,eff }}+\sigma_{S B_{\text {irr }}, \text { eff }}^{\text {int }}$ is the fiducial cross section that is accessed by the experiment. The latter can be described by folding the experimental efficiencies of each contribution in the expression of $\hat{\sigma}$ of Eqs. (1) and (4) as:

$$
\begin{aligned}
& \sigma_{\text {eff }}\left(C_{2}, M_{Q}, \Gamma_{Q} ; \chi_{Q}\right) \\
& =C_{2}^{4} \hat{\sigma}_{S}\left(M_{Q}, \Gamma_{Q}\right) \epsilon_{S}\left(M_{Q}, \Gamma_{Q}\right) \\
& \quad+C_{2}^{2} \hat{\sigma}_{S B_{\text {irr }}}^{\text {int }}\left(M_{Q}, \Gamma_{Q}, \chi_{Q}\right) \epsilon_{S B_{\text {irr }}}^{\text {int }}\left(M_{Q}, \Gamma_{Q}, \chi_{Q}\right) \\
& \equiv C_{2}^{4} \hat{\sigma}_{S, \text { eff }}\left(M_{Q}, \Gamma_{Q}\right)+C_{2}^{2} \hat{\sigma}_{S B_{\text {irr }}, \text { eff }}^{\text {iff }}\left(M_{Q}, \Gamma_{Q}, \chi_{Q}\right) .
\end{aligned}
$$

The $\hat{\sigma}_{S B_{\text {irr }}}^{\text {int }}$ plots for the interference between signal and SM background are provided in Fig. 8. Notice the different behavior in the case of dominantly $\mathrm{LH}$ or $\mathrm{RH}$ coupling 

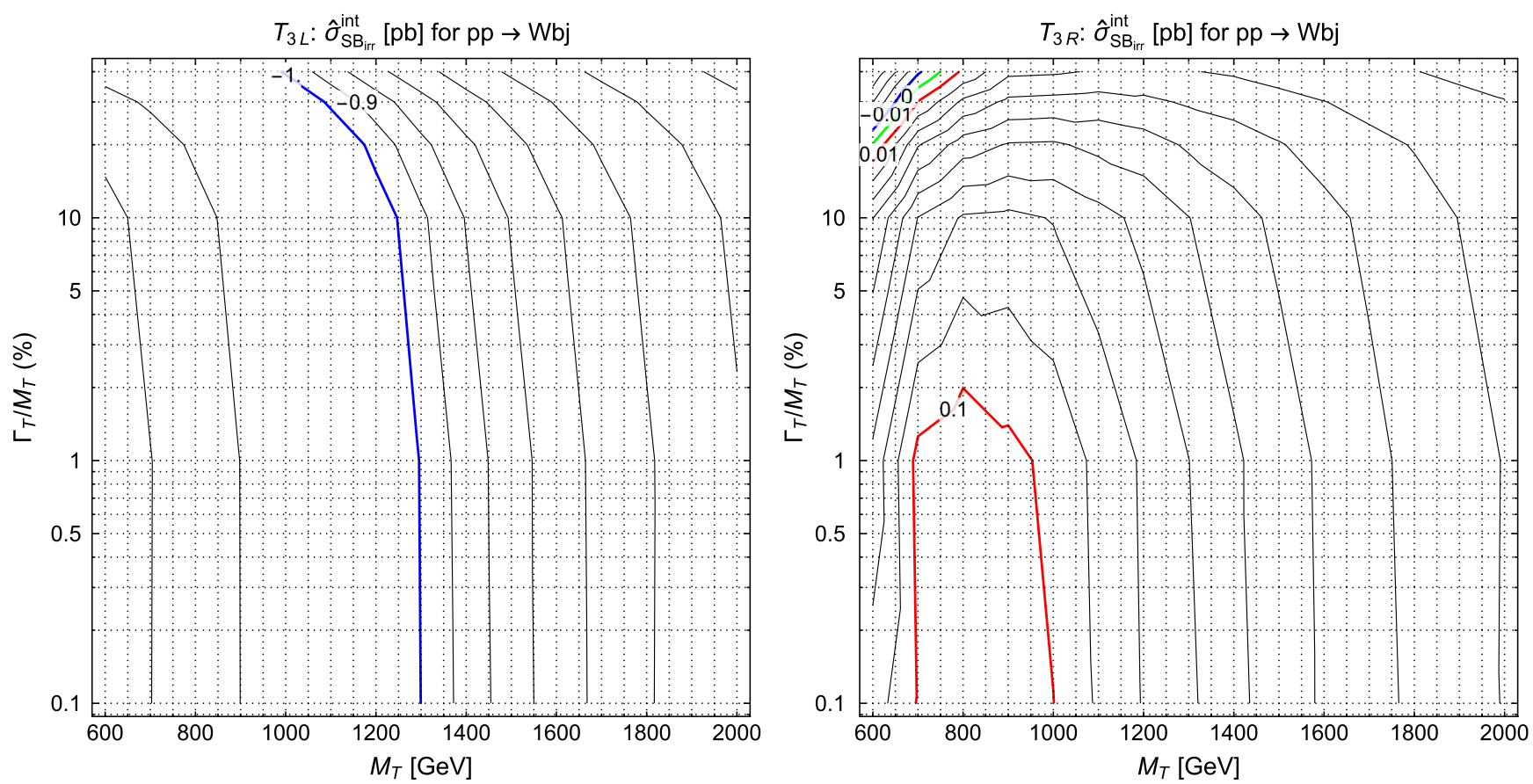

FIG. 8. $\hat{\sigma}_{S B_{\text {irr }}}^{\text {int }}$ values at $13 \mathrm{TeV}$ (in pb) for the process $p p \rightarrow b W j$ mediated by a $T$ interacting with third generation SM quarks exclusively through charged current, for the interference between signal and SM background with dominant LH (RH) coupling chirality of the $T$ in the left (right) panel.
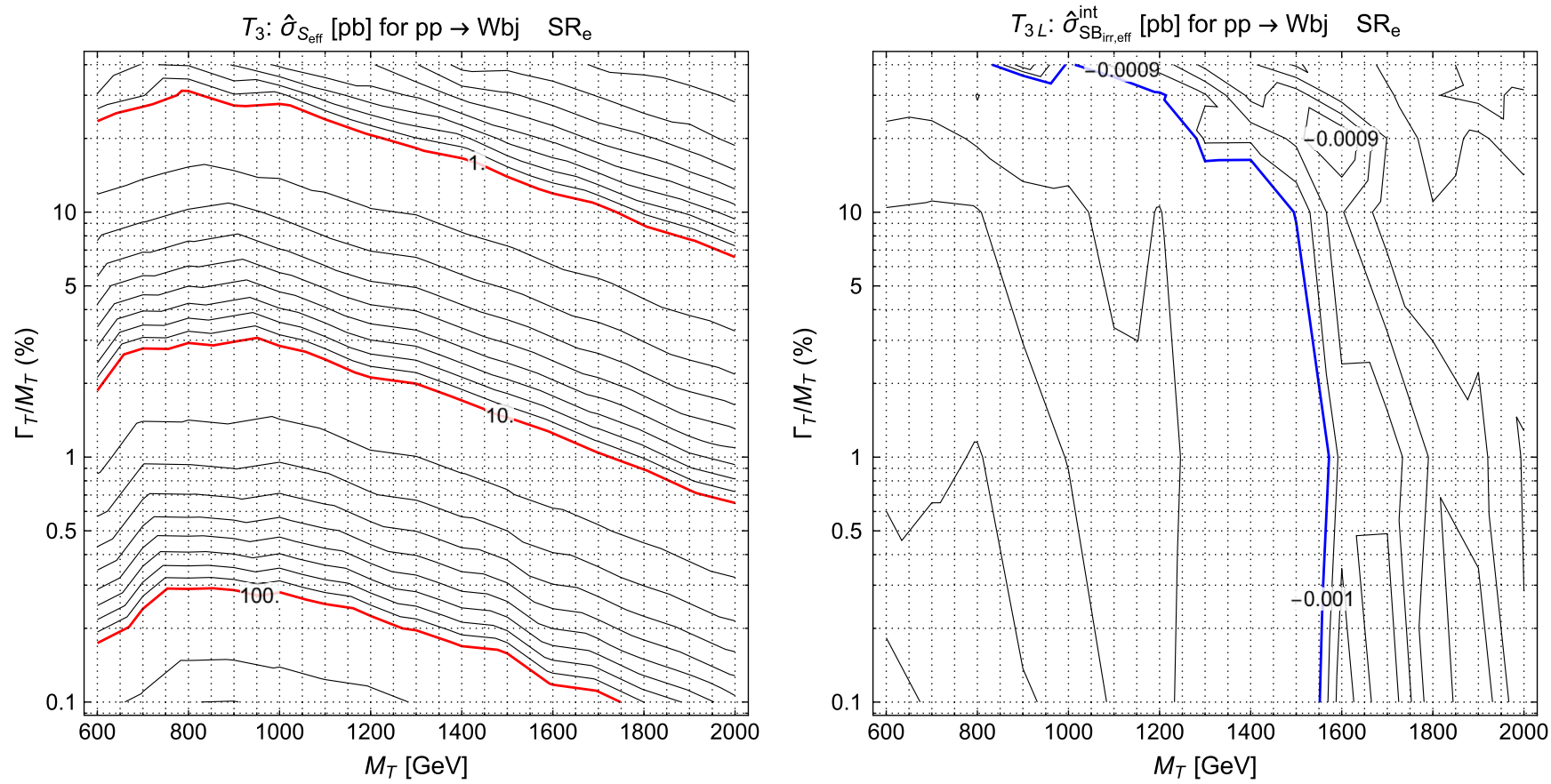

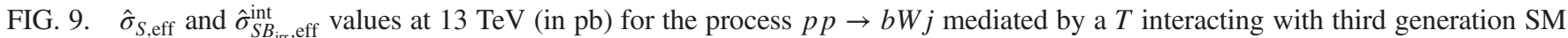
quarks exclusively through charged current, for the pure signal (left panel) and for the interference between signal and SM background with dominant LH coupling chirality of the $T$ (right panel). The signal region corresponding to the efficiencies is the single electron from the CMS search [70].

chirality of the $T$ and that, as expected, the scenario with dominant LH chirality produces larger interference contributions as the $T$ mass approaches the top mass region.
In Fig. 9 the values of $\hat{\sigma}_{S \text {,eff }}$ and $\hat{\sigma}_{S B_{\text {irr }} \text {,eff }}^{\text {int }}$ are provided for the signal and for the interference term in the case of dominant LH chirality, considering the signal region 


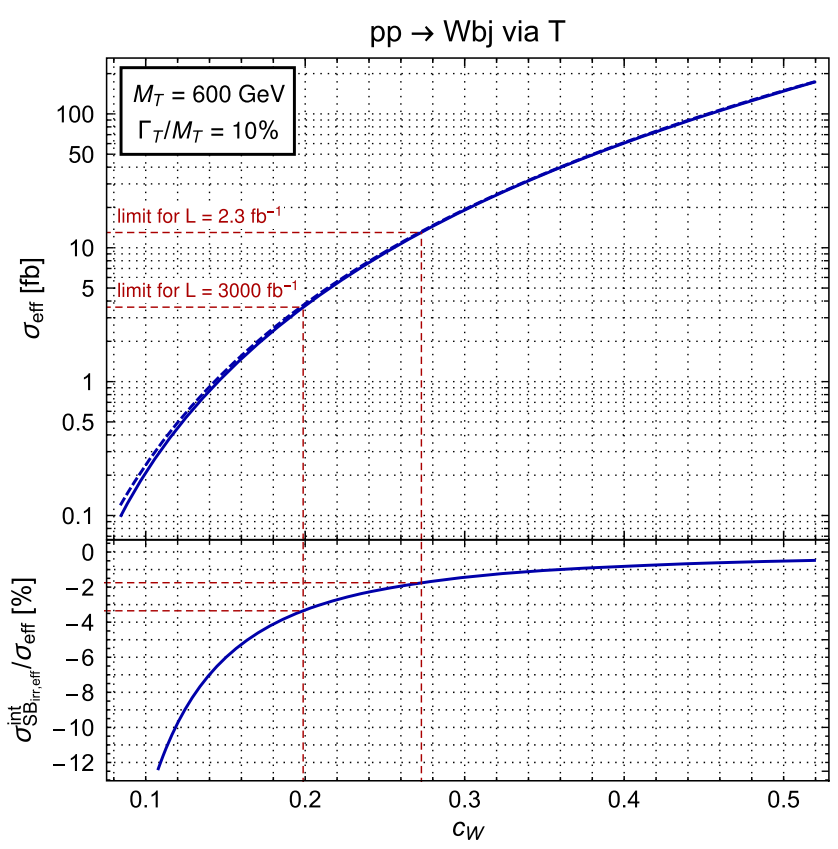

FIG. 10. Upper panel: values of $\sigma_{\text {eff }}$ with (solid line) and without (dashed line) interference contribution. Lower panel: relative contribution of the interference term to it (lower panel) at $13 \mathrm{TeV}$ as function of the $c_{W}$ coupling. The process is $p p \rightarrow b W j$ mediated by a $T$ with $M_{T}=600 \mathrm{GeV}$ and $\Gamma_{T} / M_{T}=10 \%$ interacting with third generation SM quarks exclusively through charged current. The maximum value of $c_{W}$ in the plot corresponds to a partial width equivalent to $10 \%$ of the mass. Limits at two different LHC luminosities are also shown (see text for details).

targeting final states with an electron in the CMS search [70]. With such information and with the knowledge of the total background $B$ (from experimental data), it is possible to reconstruct the number of events for $S+B$ for each point in the $\left(M_{Q}, \Gamma_{Q} / M_{Q}\right)$ plane through Eqs. (6) and (7).

To study the two scenarios described at the beginning of this section, the value of the $T W b$ coupling $c_{W}$ is modified while keeping the $\Gamma_{Q} / M_{Q}$ ratio fixed, from a maximum value which corresponds to having $\operatorname{BR}(T \rightarrow W b)=100 \%$, to smaller values which imply the existence of other decay channels. As a practical example, we consider the point with $M_{T}=600 \mathrm{GeV}$ and $\Gamma_{T} / M_{T}=10 \%$. The values of $\sigma_{\text {eff }}$ and the relative contribution of the interference term are plotted as function of the $C_{2} \equiv c_{W}$ coupling in Fig. 10, considering $c_{W}$ in a range for which $\sigma_{\text {eff }}$ is larger than $10^{-1} \mathrm{fb}$ up to when the partial width becomes equivalent to $60 \mathrm{GeV}$, (i.e., $10 \%$ of the mass). As it can also be inferred from Eq. (7), the contribution of the interference term becomes more and more relevant as the $C_{2}$ coupling decreases, eventually becoming dominant with respect to the pure signal, but the fiducial cross section $\sigma_{\text {eff }}$ decreases as well. It is now straightforward to apply a CL analysis to determine the significance for exclusion or nonexclusion of the tested point. The reported numbers of background and observed events in the electron signal region by [70] are $95 \pm 17$ and 78 respectively with a luminosity of $2.3 \mathrm{fb}^{-1}$, which imply that the quantity $\sigma_{\text {eff }} * L \simeq 30$ is excluded a $95 \% \mathrm{CL}$, or that $\sigma_{\text {eff }}$ has to be less than $13 \mathrm{fb}$. Such value places an upper bound on the $T$ coupling $c_{W} \lesssim 0.27$, in a region for which the interference contribution to the signal is around $-1.7 \%$.

The relative importance of the interference contributions becomes larger as the luminosity increases. Rescaling the number of background events for the nominal highest luminosity for the LHC, $3 \mathrm{ab}^{-1}$, we obtain $B=105556$. With such value, and assuming a systematic uncertainty for the background of 5\%, the maximum expected value of $\sigma_{\text {eff }}$ for $95 \% \mathrm{CL}$ exclusion is $3.6 \mathrm{fb}$, corresponding to $c_{W} \lesssim 0.2$, in a region for which the interference contribution to the signal is around $-3.4 \%$.

We stress that this example represents an ideal situation in which the signal and interference contributions can be factorized in a simple and gauge-invariant way. More complex scenarios, in which the VLQ can couple through neutral currents and/or other BSM states, in such a way that other interference terms are non-negligible, cannot be reinterpreted analogously to the example of this section.

\section{CONCLUSIONS}

We have studied in a model-independent way processes of single production of VLQs with non-negligible width at the LHC, exploiting a simple, yet robust, framework for the presentation of experimental results and for their subsequent reinterpretation in terms of any scenario of new physics beyond the SM which predict VLQs of generic masses, couplings and total width which can decay into SM final states. Such framework can be generalized also for different BSM scenarios or for analyses of single VLQ production where QCD NLO effects are considered.

We have assessed the role of the total width in the determination of kinematic distributions of the final state finding that, in some cases, the shapes of such distributions can significantly be deformed depending on the $\Gamma_{Q} / M_{Q}$ ratio. We have numerically recast a CMS search to determine how bounds in the plane $\left(M_{Q}, \Gamma_{Q} / M_{Q}\right)$ change and, finally, we have compared the results of the numerical recasting in one of the considered scenarios to those from the model-independent procedure, obtaining an excellent agreement. Finally we have discussed the role of interference terms, usually neglected in experimental analysis, described issues related to the reinterpretation of results if such terms are not negligible and applied a procedure for reinterpretation in an ideal scenario in which such terms can be easily factorized.

The possibility to interpret observed data in terms of exclusion regions in the parameter space of models of new physics is a crucial step for any phenomenological analysis of BSM scenarios. Nevertheless, in some cases, 
the experimental analysis techniques cannot be easily recast through phenomenological tools. In this case, for interpretations to be reliable, experimental results should provide all the essential information, so that a minimal, but complete, set of information can profitably be used for the characterization of VLQs with generic assumptions on their mass, couplings and total width. In particular, the procedure adopted here allows for the possibility to perform a reinterpretation without the need of dedicated tools, thus growing the possibilities to test scenarios of new physics predicting VLQs.

\section{ACKNOWLEDGMENTS}

S. M. is funded in part through the NExT Institute and the STFC CG ST/P000711/1.
[1] G. Aad et al. (ATLAS Collaboration), Observation of a new particle in the search for the Standard Model Higgs boson with the ATLAS detector at the LHC, Phys. Lett. B 716, 1 (2012).

[2] S. Chatrchyan et al. (CMS Collaboration), Observation of a new boson at a mass of $125 \mathrm{GeV}$ with the CMS experiment at the LHC, Phys. Lett. B 716, 30 (2012).

[3] A. Djouadi and A. Lenz, Sealing the fate of a fourth generation of fermions, Phys. Lett. B 715, 310 (2012).

[4] O. Eberhardt, G. Herbert, H. Lacker, A. Lenz, A. Menzel, U. Nierste, and M. Wiebusch, Impact of a Higgs Boson at a Mass of $126 \mathrm{GeV}$ on the Standard Model with Three and Four Fermion Generations, Phys. Rev. Lett. 109, 241802 (2012).

[5] A. Alves, E. Ramirez Barreto, D. Camargo, and A. Dias, A model with chiral quarks of electric charges $-4 / 3$ and $5 / 3$, J. High Energy Phys. 07 (2013) 129.

[6] S. Banerjee, M. Frank, and S. K. Rai, Higgs data confronts sequential fourth generation fermions in the Higgs triplet model, Phys. Rev. D 89, 075005 (2014).

[7] B. Holdom, Accidental Higgs boson, Phys. Rev. D 90, 015004 (2014).

[8] N. Bizot and M. Frigerio, Fermionic extensions of the standard model in light of the Higgs couplings, J. High Energy Phys. 01 (2016) 036.

[9] A. Davidson and K. C. Wali, Family Mass Hierarchy from Universal Seesaw Mechanism, Phys. Rev. Lett. 60, 1813 (1988).

[10] K. S. Babu and R. N. Mohapatra, Solution to the strong CP problem without an axion, Phys. Rev. D 41, 1286 (1990).

[11] B. Grinstein, M. Redi, and G. Villadoro, Low scale flavor gauge symmetries, J. High Energy Phys. 11 (2010) 067.

[12] D. Guadagnoli, R. N. Mohapatra, and I. Sung, Gauged flavor group with left-right symmetry, J. High Energy Phys. 04 (2011) 093.

[13] T. Moroi and Y. Okada, Radiative corrections to Higgs masses in the supersymmetric model with an extra family and antifamily, Mod. Phys. Lett. A 07, 187 (1992).

[14] T. Moroi and Y. Okada, Upper bound of the lightest neutral Higgs mass in extended supersymmetric standard models, Phys. Lett. B 295, 73 (1992).

[15] K. S. Babu, I. Gogoladze, M. U. Rehman, and Q. Shafi, Higgs boson mass, sparticle spectrum and little hierarchy problem in extended MSSM, Phys. Rev. D 78, 055017 (2008).
[16] S. P. Martin, Extra vector-like matter, and the lightest Higgs scalar boson mass in low-energy supersymmetry, Phys. Rev. D 81, 035004 (2010).

[17] P. W. Graham, A. Ismail, S. Rajendran, and P. Saraswat, Little solution to the little hierarchy problem: A vectorlike generation, Phys. Rev. D 81, 055016 (2010).

[18] S. P. Martin, Raising the Higgs mass with Yukawa couplings for isotriplets in vector-like extensions of minimal supersymmetry, Phys. Rev. D 82, 055019 (2010).

[19] J. L. Rosner, E6 and exotic fermions, Comments Nucl. Part. Phys. 15, 195 (1986).

[20] R. Robinett, On the mixing and production of exotic fermions in E6, Phys. Rev. D 33, 1908 (1986).

[21] N. Arkani-Hamed, A. Cohen, E. Katz, and A. Nelson, The littlest Higgs, J. High Energy Phys. 07 (2002) 034.

[22] M. Schmaltz and D. Tucker-Smith, Little higgs theories, Annu. Rev. Nucl. Part. Sci. 55, 229 (2005).

[23] B. A. Dobrescu and C. T. Hill, Electroweak Symmetry Breaking via a Top Condensation Seesaw Mechanism, Phys. Rev. Lett. 81, 2634 (1998).

[24] R. S. Chivukula, B. A. Dobrescu, H. Georgi, and C. T. Hill, Top quark seesaw theory of electroweak symmetry breaking, Phys. Rev. D 59, 075003 (1999).

[25] H.-J. He, C. T. Hill, and T. M. Tait, Top quark seesaw, vacuum structure and electroweak precision constraints, Phys. Rev. D 65, 055006 (2002).

[26] C. T. Hill and E. H. Simmons, Strong dynamics and electroweak symmetry breaking, Phys. Rep. 381, 235 (2003).

[27] K. Agashe, R. Contino, and A. Pomarol, The minimal composite Higgs model, Nucl. Phys. B719, 165 (2005).

[28] R. Contino, L. Da Rold, and A. Pomarol, Light custodians in natural composite Higgs models, Phys. Rev. D 75, 055014 (2007).

[29] R. Barbieri, B. Bellazzini, V. S. Rychkov, and A. Varagnolo, Higgs boson from an extended symmetry, Phys. Rev. D 76, 115008 (2007).

[30] C. Anastasiou, E. Furlan, and J. Santiago, Realistic composite Higgs models, Phys. Rev. D 79, 075003 (2009).

[31] J. Aguilar-Saavedra, Identifying top partners at LHC, J. High Energy Phys. 11 (2009) 030.

[32] Y. Okada and L. Panizzi, LHC signatures of vector-like quarks, Adv. High Energy Phys. 2013, 1 (2013).

[33] A. De Simone, O. Matsedonskyi, R. Rattazzi, and A. Wulzer, A first top partner Hunter's guide, J. High Energy Phys. 04 (2013) 004. 
[34] M. Buchkremer, G. Cacciapaglia, A. Deandrea, and L. Panizzi, Model independent framework for searches of top partners, Nucl. Phys. B876, 376 (2013).

[35] J. A. Aguilar-Saavedra, R. Benbrik, S. Heinemeyer, and M. Pérez-Victoria, Handbook of vectorlike quarks: Mixing and single production, Phys. Rev. D 88, 094010 (2013).

[36] M. Aaboud et al. (ATLAS Collaboration), Search for pair production of vector-like top quarks in events with one lepton, jets, and missing transverse momentum in $\sqrt{s}=$ $13 \mathrm{TeV} p p$ collisions with the ATLAS detector, J. High Energy Phys. 08 (2017) 052.

[37] M. Aaboud et al. (ATLAS Collaboration), Search for pair production of heavy vector-like quarks decaying to high- $p_{T}$ $\mathrm{W}$ bosons and $\mathrm{b}$ quarks in the lepton-plus-jets final state in pp collisions at $\sqrt{s}=13 \mathrm{TeV}$ with the ATLAS detector, J. High Energy Phys. 10 (2017) 141.

[38] M. Aaboud et al. (ATLAS Collaboration), Search for pair production of up-type vector-like quarks and for four-topquark events in final states with multiple $b$-jets with the ATLAS detector, arXiv:1803.09678.

[39] A. M. Sirunyan et al. (CMS Collaboration), Search for pair production of vector-like $\mathrm{T}$ and $\mathrm{B}$ quarks in single-lepton final states using boosted jet substructure in proton-proton collisions at $\sqrt{s}=13 \mathrm{TeV}$, J. High Energy Phys. 11 (2017) 085 .

[40] A. M. Sirunyan et al. (CMS Collaboration), Search for pair production of vector-like quarks in the $\mathrm{bW} \bar{b} \mathrm{~W}$ channel from proton-proton collisions at $\sqrt{s}=13 \mathrm{TeV}$, Phys. Lett. B 779, 82 (2018).

[41] A. M. Sirunyan et al. (CMS Collaboration), Search for vector-like $\mathrm{T}$ and $\mathrm{B}$ quark pairs in final states with leptons at $\sqrt{s}=13 \mathrm{TeV}$, arXiv:1805.04758.

[42] A. M. Sirunyan et al. (CMS Collaboration), Search for single production of a vector-like $\mathrm{T}$ quark decaying to a $\mathrm{Z}$ boson and a top quark in proton-proton collisions at $\sqrt{s}=13$ TeV, Phys. Lett. B 781, 574 (2018).

[43] A. M. Sirunyan et al. (CMS Collaboration), Search for single production of vector-like quarks decaying to $a b$ quark and a Higgs boson, J. High Energy Phys. 06 (2018) 031.

[44] ATLAS Collaboration, Search for single production of vectorlike quarks decaying into $W b$ in $p p$ collisions at $\sqrt{s}=$ $13 \mathrm{TeV}$ with the ATLAS detector, Report No. ATLASCONF-2016-072, 2016, http://inspirehep.net/record/ 1480052.

[45] D. Barducci, A. Belyaev, M. Buchkremer, G. Cacciapaglia, A. Deandrea, S. De Curtis, J. Marrouche, S. Moretti, and L. Panizzi, Framework for model independent analyses of multiple extra quark scenarios, J. High Energy Phys. 12 (2014) 080.

[46] D. Barducci, A. Belyaev, M. Buchkremer, J. Marrouche, S. Moretti, and L. Panizzi, XQCAT: eXtra Quark Combined Analysis Tool, Comput. Phys. Commun. 197, 263 (2015).

[47] M. Chala, Direct bounds on heavy toplike quarks with standard, and exotic decays, Phys. Rev. D 96, 015028 (2017).

[48] M. Chala, R. Grber, and M. Spannowsky, Searches for vector-like quarks at future colliders and implications for composite Higgs models with dark matter, J. High Energy Phys. 03 (2018) 040.
[49] D. Barducci, A. Belyaev, J. Blamey, S. Moretti, L. Panizzi, and H. Prager, Towards model-independent approach to the analysis of interference effects in pair production of new heavy quarks, J. High Energy Phys. 07 (2014) 142.

[50] S. Moretti, D. O’Brien, L. Panizzi, and H. Prager, Production of extra quarks at the Large Hadron Collider beyond the narrow width approximation, Phys. Rev. D 96, 075035 (2017).

[51] S. Moretti, D. O'Brien, L. Panizzi, and H. Prager, Production of extra quarks decaying to dark matter beyond the narrow width approximation at the LHC, Phys. Rev. D 96, 035033 (2017).

[52] H. Prager, S. Moretti, D. O'Brien, and L. Panizzi, Extra quarks decaying to dark matter beyond the narrow width approximation, in 5th Large Hadron Collider Physics Conference (LHCP 2017) Shanghai, China, 2017, 2017 (http://inspirehep.net/record/1604905/files/arXiv:1706.04001 .pdf).

[53] H. Prager, S. Moretti, D. O’Brien, and L. Panizzi, Large width effects in processes of production of extra quarks decaying to Dark Matter at the LHC, in 25th International Workshop on Deep-Inelastic Scattering and Related Topics (DIS 2017) Birmingham, UK, 2017, 2017 (http://inspirehep .net/record/1604906/files/arXiv:1706.04007.pdf).

[54] C.-Y. Chen, S. Dawson, and E. Furlan, Vectorlike fermions and Higgs effective field theory revisited, Phys. Rev. D 96, 015006 (2017).

[55] G. Cacciapaglia, A. Deandrea, N. Gaur, D. Harada, Y. Okada, and L. Panizzi, Interplay of vector-like top partner multiplets in a realistic mixing set-up, J. High Energy Phys. 09 (2015) 012.

[56] J. M. Campbell, R. Frederix, F. Maltoni, and F. Tramontano, Next-to-Leading-Order Predictions for t-Channel SingleTop Production at Hadron Colliders, Phys. Rev. Lett. 102, 182003 (2009).

[57] R. Frederix, E. Re, and P. Torrielli, Single-top t-channel hadroproduction in the four-flavour scheme with POWHEG and aMC@NLO, J. High Energy Phys. 09 (2012) 130.

[58] E. Bothmann, F. Krauss, and M. Schnherr, Single top-quark production with SHERPA, Eur. Phys. J. C 78, 220 (2018).

[59] G. Brooijmans et al., Les Houches 2017: Physics at TeV Colliders New Physics Working Group Report, in 10th Les Houches Workshop on Physics at TeV Colliders (PhysTeV 2017) Les Houches, France, 2017, 2018 (https://inspirehep .net/record/1664565/files/1803.10379.pdf).

[60] J. Alwall, M. Herquet, F. Maltoni, O. Mattelaer, and T. Stelzer, MadGraph 5: Going beyond, J. High Energy Phys. 06 (2011) 128.

[61] J. Alwall, R. Frederix, S. Frixione, V. Hirschi, F. Maltoni, O. Mattelaer, H.-S. Shao, T. Stelzer, P. Torrielli, and M. Zaro, The automated computation of tree-level and next-to-leading order differential cross sections, and their matching to parton shower simulations, J. High Energy Phys. 07 (2014) 079.

[62] R. D. Ball et al. (NNPDF Collaboration), Parton distributions for the LHC Run II, J. High Energy Phys. 04 (2015) 040.

[63] T. Sjostrand, S. Mrenna, and P.Z. Skands, A brief introduction to PYTHIA 8.1, Comput. Phys. Commun. 178, 852 (2008).

[64] T. Sjostrand, S. Mrenna, and P.Z. Skands, PYTHIA 6.4 physics and manual, J. High Energy Phys. 05 (2006) 026. 
[65] E. Conte, B. Dumont, B. Fuks, and C. Wymant, Designing and recasting LHC analyses with MadAnalysis 5, Eur. Phys. J. C 74, 3103 (2014).

[66] J. de Favereau, C. Delaere, P. Demin, A. Giammanco, V. Lemaître, A. Mertens, and M. Selvaggi (DELPHES 3 Collaboration), DELPHES 3, A modular framework for fast simulation of a generic collider experiment, J. High Energy Phys. 02 (2014) 057.

[67] A. Alloul, N. D. Christensen, C. Degrande, C. Duhr, and B. Fuks, FeynRules 2.0-A complete toolbox for tree-level phenomenology, Comput. Phys. Commun. 185, 2250 (2014).
[68] C. Degrande, C. Duhr, B. Fuks, D. Grellscheid, O. Mattelaer, and T. Reiter, UFO-The universal FeynRules output, Comput. Phys. Commun. 183, 1201 (2012).

[69] B. Fuks and H.-S. Shao, QCD next-to-leading-order predictions matched to parton showers for vector-like quark models, Eur. Phys. J. C 77, 135 (2017).

[70] A. M. Sirunyan et al. (CMS Collaboration), Search for single production of vector-like quarks decaying into $a b$ quark and a $\mathrm{W}$ boson in proton-proton collisions at $\sqrt{s}=13 \mathrm{TeV}$, Phys. Lett. B 772, 634 (2017). 